\title{
Advanced care planning in the elderly, are we doing it?
}

\author{
Authors: Towhid Imam, Teuny Jones, Sunday Afolayan and Shilpa Raje
}

\section{Aims}

The aim was to highlight that elderly patients nearing the end of their life should have an advanced care plan (ACP) as per national guidelines. This helps patients approaching the end of their life to plan for their future care and affairs. This should be discussed before patients become unwell and in the community while they have mental capacity.

\section{Methods}

The National Gold Standards Framework (GSF) for ACP was used on three wards for older people. The criteria measured were:

$>$ general indicators of decline and increasing needs

$>$ signs of frailty

$>$ signs of late-stage dementia.

\section{Results}

27 cases were examined:

$>56 \%$ had five or more general indicators

$>85 \%$ had three or more out of six signs of frailty

$>89 \%$ had two or more signs of late-stage dementia

$>41 \%$ were discharged without mention of care planning and $45 \%$ of these had multiple readmissions

$>33 \%$ had palliative discharges and $15 \%$ died in hospital

$>$ None of the patients had an ACP from the community.

\section{Conclusions}

ACP aids in planning for future care and a better patient experience. Our study has shown the practice of ACP needs to be improved in patients with poor prognostic indicators. Care plans in hospital and in the community could potentially be clearer and more integrated. One possible solution is to use online platforms to store this information. It is the responsibility of all clinicians at all levels to encourage and participate in ACP. 